\title{
Poly-L-Lactic Acid Nanotubes as Soft Piezoelectric Interfaces for Biology: Controlling Cell Attachment via Polymer Crystallinity
}

\author{
Michael Smith, Thomas Chalklen, Cathrin Lindackers, Yonatan Calahorra, Caitlin Howe, \\ Alkausil Tamboli, Daniel V. Bax, David J. Barrett, Ruth E. Cameron, Serena M. Best, \\ and Sohini Kar-Narayan*
}

Cite This: ACS Appl. Bio Mater. 2020, 3, 2140-2149

Read Online

\section{ACCESS \\ 山ll Metrics \& More \\ Article Recommendations \\ Supporting Information}

ABSTRACT: It has become increasingly evident that the mechanical and electrical environment of a cell is crucial in determining its function and the subsequent behavior of multicellular systems. Platforms through which cells can directly interface with mechanical and electrical stimuli are therefore of great interest. Piezoelectric materials are attractive in this context because of their ability to interconvert mechanical and electrical energy, and piezoelectric nanomaterials, in particular, are ideal candidates for tools within mechanobiology, given their ability to both detect and apply small forces on a length scale that is compatible with cellular dimensions. The choice of piezoelectric
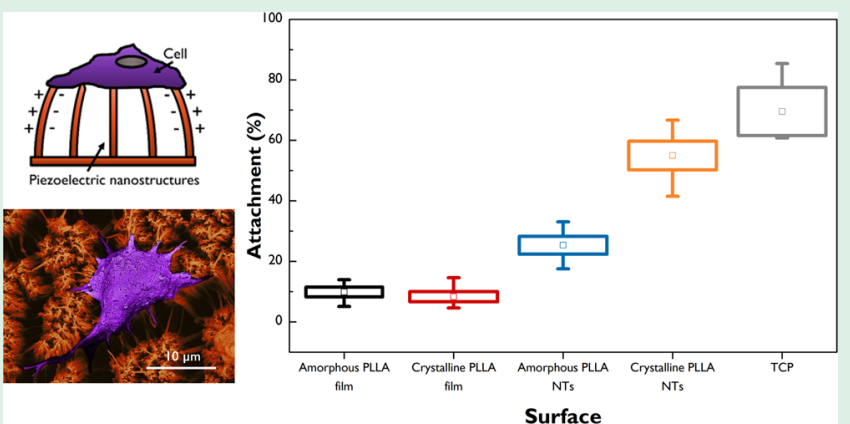
material is crucial to ensure compatibility with cells under investigation, both in terms of stiffness and biocompatibility. Here, we show that poly-L-lactic acid nanotubes, grown using a melt-press template wetting technique, can provide a "soft" piezoelectric interface onto which human dermal fibroblasts readily attach. Interestingly, by controlling the crystallinity of the nanotubes, the level of attachment can be regulated. In this work, we provide detailed nanoscale characterization of these nanotubes to show how differences in stiffness, surface potential, and piezoelectric activity of these nanotubes result in differences in cellular behavior.

KEYWORDS: mechanobiology, polymer nanotubes, piezoelectric polymer, poly-L-lactic acid, fibroblasts, cell attachment

\section{INTRODUCTION}

Cells are naturally exposed to a wealth of stimuli that influence their function and behavior. The chemical aspect of this signaling has been recognized for centuries, yet it is only in the past few decades that the mechanical and electrical sensitivity of biological systems has become apparent. The mechanical environment of cellular systems can regulate the shape and function of many cell lines ${ }^{1,2}$ and even guide the fate of stem cell differentiation. ${ }^{3,4}$ Electrical stimulation of cells has also been shown to influence a number of biological processes in vitro including cell attachment, cell division, and cell movement, as well as bone production and wound healing in vivo. ${ }^{5}$ Observations of these phenomena have fueled significant interest in the emerging fields of mechanobiology ${ }^{6-9}$ and bioelectronics. ${ }^{10-13}$ This attention is motivated partly by academic curiosity but also because of the exciting prospect of an entirely new perspective on the treatment and management of diseases. The pharmaceutical industry is dependent on the chemical sensitivity of biological systems, but as modern medicine advances into tissue engineering and regenerative medicine, it is vital that all aspects of biological signalingchemical, mechanical, and electrical-are understood and controlled. As a result, there is currently great interest in designing devices and environments that can exploit the electromechanical sensitivity of cells. ${ }^{14,15}$ In particular, there has recently been a huge increase in the popularity of piezoelectric materials for cell culture applications. The inherent coupling between mechanical and electrical properties is interesting from an electromechanical stimulation perspective, as is the fact that many biological materials, including wood, bone, tendon, skin, and DNA, ${ }^{16-19}$ are themselves piezoelectric.

The use of piezoelectric materials in cell culture applications is discussed at length in a number of recent reviews, all published in the past three years. ${ }^{20-27}$ Typically, cells are cultured directly onto scaffolds made from piezoelectric ceramics, ${ }^{26,28,29}$ piezoelectric polymers, ${ }^{30-33}$ or polymer/ceramic composites where one or both components may be piezoelectric. ${ }^{34,35}$ In many cases, the cell culture conditions are static, that is, no additional

Received: January 4, 2020

Accepted: March 11, 2020

Published: March 11, 2020 
mechanical or electrical stimulation is applied to the scaffold during incubation. As Tandon et al. mention in their recent review, ${ }^{24}$ there is a significant problem with this protocol. The rationale for using piezoelectric materials is that any mechanical stimulation of the cell culture is also coupled to electrical stimulation. If mechanical stimulation is absent, then so too is any electrical stimulation. Dynamic conditions are possible but require an external transducer to provide some mechanical perturbation. ${ }^{32,36,37}$ For some applications, such as those which require implantation, this is not a valid approach.

Achieving dynamic culture conditions without an external transducer requires the cells themselves to deform the piezoelectric material. While cells are capable of exerting traction forces to their surroundings, these are typically of the order of pico- to nanonewtons. ${ }^{38}$ Achieving piezoelectric stimulation under these quasistatic cell culture conditions requires a piezoelectric material that is suitably "soft". As shown in Figure 1a, ${ }^{39-42}$ "soft" piezoelectric materials do not exist, at least within the range of moduli typically found in

(a)

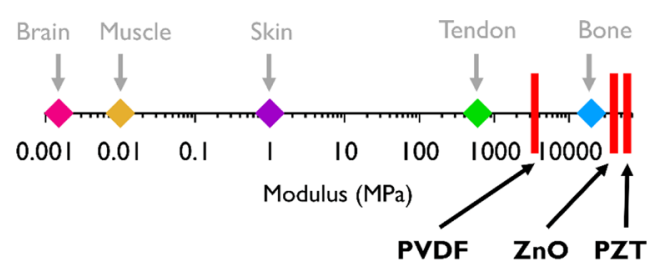

(b)

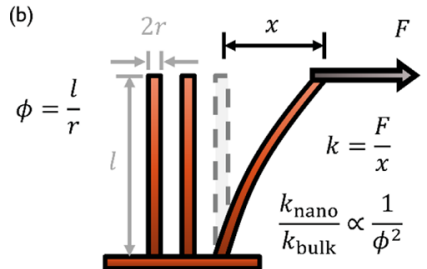

(d)

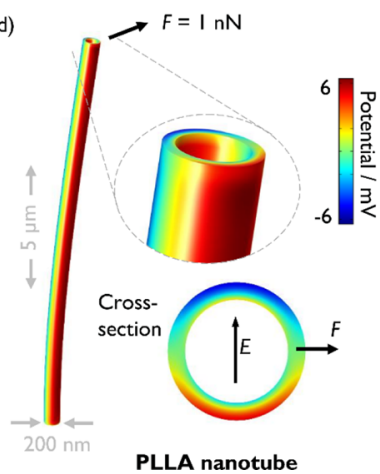

(c)

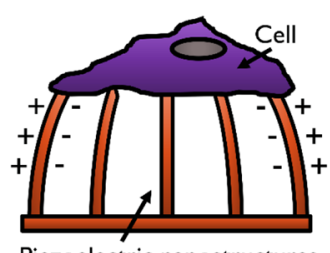

Piezoelectric nanostructures

(e)

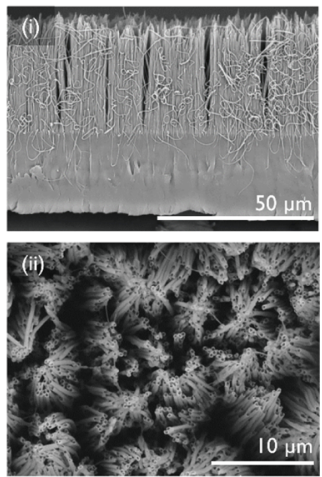

Figure 1. (a) Schematic representation of the broad range of stiffness in the biological tissue and how these compare to the moduli of typical piezoelectric materials: lead zirconium titanate (PZT), zinc oxide $(\mathrm{ZnO})$, and poly(vinylidene fluoride). (b) Schematic of the bending mode of high aspect ratio structures with aspect ratio $\phi$, which results in reduced effective stiffness $k_{\text {nano }}$ by a factor of $\phi^{-2}$ with respect to the bulk material. (c) Proposed mechanism by which a cell can electromechanically stimulate itself by interacting with the piezoelectric nanostructures. (d) Simulation of a PLLA nanotube with axial polymer chain orientation, showing the potential developed in response to bending. Inset shows the opposing potentials developed across the tube diameter and the orientation of the corresponding electric field. (e) An example of the PLLA nanotube arrays produced via melt-press template wetting (i) in cross-section and (ii) in plan view. biological tissue, and therefore these traction forces are insufficient to induce any significant strain (and therefore polarization) in conventional bulk piezoelectric materials. There is very little scope to alter the intrinsic modulus of piezoelectric materials. Instead, to address the issue of stiffness, the extrinsic compliance of the structure can be modified. Nanostructures can have exceptionally high aspect ratios and as a result can be very susceptible to bending modes of deformation. An array of vertically aligned nanostructures therefore appears significantly softer than the bulk material with respect to in-plane shear deformation, as demonstrated in Figure 1b (see Supporting Information S1 for further details).

In this work, we demonstrate that high-aspect ratio nanostructures can be used to create "soft" piezoelectric surfaces which can directly interface with growing cells, as schematically outlined in Figure 1c. Similar approaches have been attempted before but with inorganic, nonbiocompatible piezoelectric materials. ${ }^{43}$ Here, we show that nanotubes of the piezoelectric bio-polymer poly-L-lactic acid (PLLA) are ideally suited to this application. The piezoelectric properties of this polymer, combined with straightforward nanofabrication methods, result in flexible, biocompatible and biodegradable piezoelectric nanotubes that develop significant material polarization in response to bending. Human dermal fibroblast (HDF) cells cultured on these surfaces are therefore able to electromechanically stimulate themselves simply by interacting with their environment. Furthermore, crystallization of the polymer nanostructures allows for the electromechanical properties of the surface to be tuned. Previous studies on PLLA, both in the bulk and in nanofiber mats, have shown that crystallinity can influence cell response, although the mechanism is not well understood. ${ }^{44-47}$ However in the present work, an explanation is given for the influence of nanostructure crystallinity on cell attachment. This work is therefore the first demonstration of "soft" piezoelectric surfaces for biological applications with tunable electromechanical properties.

\section{RESULTS AND DISCUSSION}

PLLA Nanotubes as Flexible Piezoelectric Structures. In order to produce a soft piezoelectric surface via nanostructuring, it is reasonable to start with a bulk piezoelectric material that is already somewhat compliant. Piezoelectric polymers are interesting materials in this regard, with elastic moduli typically an order of magnitude lower than highperformance piezoelectric ceramics (albeit with a similar decrease in piezoelectric coefficients). ${ }^{48}$ PLLA stands out for this particular application because of its biological credentials ${ }^{49}$ and its shear piezoelectric properties. ${ }^{50}$ PLLA is already widely used in biomedicine because of its biodegradable and biocompatible properties. ${ }^{51}$ Bone fixings and tissue scaffolds made from PLLA can be implanted to support the healing tissue before degrading into lactic acid and being resorbed by the body. ${ }^{52}$ Under appropriate conditions, PLLA is also piezoelectric, although the implications of this for biomedical applications are yet to be fully explored..$^{53-56}$ PLLA exhibits shear piezoelectricity, that is, the non-zero components of the piezoelectric tensor (in Voigt notation) are $d_{14}=-d_{25}$. Typically $d_{14}$ is measured to be around $10 \mathrm{pC} / \mathrm{N},{ }^{23,25,50}$ although the exact value is dependent on material processing.

The component $d_{14}$ couples a shear stress to material polarization (and vice versa). This is useful when considering the bending mode of deformation proposed above. The shear force in a rigidly fixed, end-loaded cantilever is roughly constant 


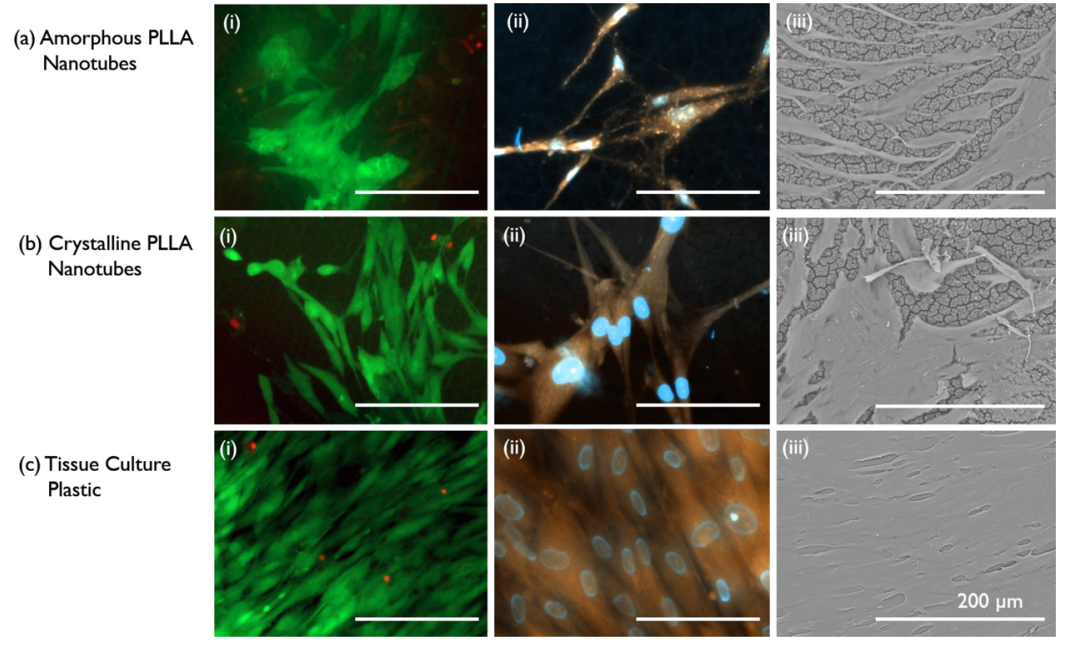

Figure 2. Cellular interaction with PLLA nanotubes. All images were taken after $72 \mathrm{~h}$ culture. HDFs grown on (a) the amorphous PLLA nanotube surface. (b) Crystalline PLLA nanotube surface. (c) TCP. (i) Live/dead staining of HDFs, live cells shown in green, dead cells in red. (ii) Rhodamine and DAPI staining of HDFs, demonstrating clustering on nanotubes, in contrast to elongation on TCP (iii) SEM images of HDFs showing coverage and surface morphology.

along its length. A nanostructure made from PLLA would therefore generate piezoelectric potential along its entire length when bent, provided that the strain from bending couples to the non-zero elements of $d_{i j}$. This is achieved by considering the orientation of the piezoelectric material, which in polymers is determined by the alignment of the polymer chains. For PLLA, with non-zero $d_{14}=-d_{25}$, an axial polymer chain orientation would result in a significant diametric potential when the nanostructure is bent (see Supporting Information S2).

Previous work in this group has demonstrated that melt-press template wetting of PLLA results in arrays of vertically aligned nanotubes with an axial polymer chain orientation, ${ }^{57}$ exactly as required for the "soft" piezoelectric surface outline above. Finite element analysis (FEA) of this structure shown in Figure 1d was used to validate that piezoelectric potential is indeed developed in response to bending. Opposing potentials were found to develop on the either side of the nanotube; importantly, this also applies to the very end of the structure, where the force is applied. On the other hand, in piezoelectric polymers which operate with normal (as opposed to shear) piezoelectric coefficients, the maximum potential can occur away from the point of force application, that is, away from the cell attachment site. With PLLA nanotubes, an attached cell would experience an in-plane electric field of the order of $10^{4} \mathrm{~V} / \mathrm{m}$ (Figure 1d).

The melt-press template wetting method results in nanotubes $\approx 30 \mu \mathrm{m}$ long, $\approx 300 \mathrm{~nm}$ in diameter, and a wall thickness of $\approx 50 \mathrm{~nm}$. The surface and cross-section are shown in Figure 1e. Analysis of how the tube length, radius, and wall thickness influence the surface potential in response to bending can be found in Figure S3 of the Supporting Information. The length of these nanotubes is significantly greater than those modeled with FEA $(30 \mu \mathrm{m} v s 5 \mu \mathrm{m})$. It is very computationally intensive to accurately model the entire length of the observed nanotubes; however, the trends and insights provided by modeling the shorter tubes can be extrapolated and applied to the longer tubes observed experimentally.

HDF Response to Soft PLLA Nanotube-Based Piezoelectric Surfaces. SEM images of HDFs cultured on these nanostructured surfaces are shown in Figure $2 \mathrm{a}(\mathrm{iii}), \mathrm{b}(\mathrm{iii})$. Further images are shown in the Supporting Information Figure S5. The nanotubes have a tendency to cluster and clump together, even in the absence of cells. However, there is evidence to suggest that HDFs cultured on the nanotube surface are capable of deforming the nanotubes within each cluster. It should also be noted that these SEM images are obtained after dehydrating the samples, so some drying artefacts may be present.

The behavior of HDFs on the nanotube surface is different in a subtle way to that observed on standard tissue culture plastic (TCP). Figure $2 c$ demonstrates how HDFs on TCP appear more orientated, and distinct, while those on the PLLA NTs are more clustered and less elongated, as shown in Figure 2a,b. Live/ dead staining of cells grown on these surfaces for $72 \mathrm{~h}$-as shown in Figure $2 a(i), b(i)$-indicates that the material and structures are biocompatible. This is somewhat unsurprising, given the biological credentials of PLLA. PLLA is a biodegradable polymer and will degrade over a timescale of weeks and months. ${ }^{58}$ The cell testing in this study was performed at shorter timescales to mitigate the influence of material degradation. ${ }^{46}$

The geometry of the nanotubes results in an effective stiffness approximately $10^{4}$ times lower than that of bulk PLLA, with respect to in-plane shear deformation (as calculated using the method outlined in Supporting Information S1). Using the observed values of length, radius, and wall thickness, and assuming a Young's modulus of $4 \mathrm{GPa}^{49}{ }^{49}$ the bending stiffness of a single nanotube is calculated to be $0.32 \mathrm{nN} / \mu \mathrm{m}-$ (see Supporting Information S6 for details). However, as shown in the images in Figure S5, the cells do not interact with single nanotubes. Instead, the nanotubes cluster together and are deformed as a group. The calculated value for stiffness can therefore only be considered as a lower bound.

An absolute upper bound on the stiffness can be calculated by modeling each clump of nanotubes as a single nanotube with a diameter equal to that of the group. This analysis gives the upper bound as $200 \mathrm{nN} / \mu \mathrm{m}$. These values are in a similar range to those reported elsewhere for micropost arrays. ${ }^{3}$

It is also interesting to calculate an equivalent shear modulus for this surface; that is, for the same thickness of some equivalent bulk material, what value of shear modulus would be required in order for the force per unit lateral displacement at the surface to be the same. Using the same lower and upper bound approach, 
the equivalent shear modulus is between $220 \mathrm{kPa}$ and 5.4 MPa. The actual value of stiffness and equivalent shear modulus will be much closer to the lower bound, but these estimates serve to put the mechanics of this surface in context with other biologically relevant soft materials.

In the previously mentioned work regarding melt-pressed PLLA nanotubes, ${ }^{57}$ it was also shown that heat treatment can be used to induce crystallization in the nanostructures. The crystallinity of polymeric materials can have a significant influence of their physical, chemical, and electrical properties. ${ }^{59}$ Thus, it is interesting to observe the response of HDFs to PLLA nanotubes of different crystalline fractions. Amorphous and crystalline PLLA nanotubes were therefore prepared for cell culture, with crystalline samples displaying an average crystalline (volume) fraction of $\approx 50 \%$.

Figure 3 a shows how the percentage cell attachment varies between arrays of amorphous and crystalline PLLA nanotubes,

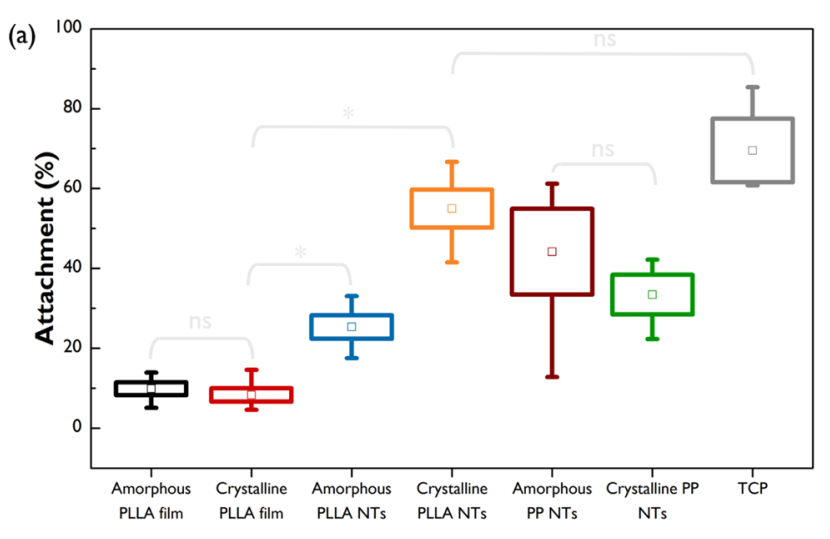

Surface

(b)

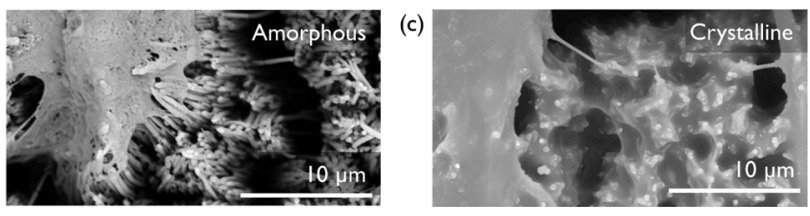

Figure 3. (a) Percentage cell attachment of HDFs to various different surfaces, as measured by a LDH assay after $14 \mathrm{~h}$. Apart from TCP, none of the surfaces were pretreated. Significance assessed via a $t$-test; ns = not significant; $*=p<0.01$. Square indicates mean value, box represents standard error in mean, whiskers show min. \& max. values. (b) SEM image of HDF attachment to amorphous PLLA nanotubes. (c) SEM image of a substance coating on crystalline PLLA nanotubes believed to be an extra-cellular matrix. This coating was only observed on crystalline samples.

flat films of amorphous and crystalline PLLA, arrays of nonpiezoelectric amorphous and crystalline polypropylene (PP), and standard TCP for reference. The stiffness of the PLLA films changed upon crystallization (see Supporting Information S7), but neither were found to be piezoelectric because no overall molecular alignment existed in either sample. PP is a semi-crystalline but nonpiezoelectric polymer, and it is thus useful to highlight the influence of piezoelectricity on cell growth. It is also important to note that none of the samples were pretreated (aside from TCP plasma treatment) or precoated with any adhesive factors in the cell culture experiments.

It is clear that crystalline nanotubes were the most adherent PLLA surface, with an average of $55 \%$ of the applied cells adhering to the surface. This approached the attachment to $\mathrm{TCP}$, considered as a standard cell culture surface. Indeed, the differences in attachment observed between crystalline nanotubes and TCP in this experiment were not found to be statistically significant at a 0.05 level. Figure $3 b, c$ displays SEM images of HDF cells cultured for $72 \mathrm{~h}$ on both amorphous and crystalline PLLA nanotubes, respectively. A substance can be seen coating the crystalline nanotubes in the vicinity of the cells. It is possible that this layer is an extracellular matrix (ECM) excreted by the cells in response to their surroundings. Clearly, crystallizing the PLLA nanotubes altered the properties of the nanostructures such that the cellular response was changed. Crystallization in PP nanotubes, however, resulted in no significant difference in the cellular behavior.

Nanoscale Characterisation of PLLA Nanotubes. It is clear that the interaction between cell and nanotube essentially occurs at the nanoscale. To fully understand exactly how and why polymer crystallization affects cellular response, it was necessary to characterize the nanotubes at a similar length scale. Various scanning probe microscopy (SPM) methods have therefore been used to understand how crystallization changes the properties of PLLA nanotubes at the nanoscale. Kelvin probe force microscopy (KPFM), ${ }^{60}$ quantitative nanomechanical mapping (QNM), ${ }^{61}$ and piezoresponse force microscopy $(\mathrm{PFM})^{62}$ have been used to characterize the surface potential, mechanical properties, and piezoelectric response, respectively, of both amorphous and crystalline PLLA nanotubes. These properties are known to be a function of crystallinity in bulk polymer samples, ${ }^{63-65}$ but demonstrating the consequences of crystallization at the nanoscale is somewhat less well explored.

Figure 4 shows a summary of the (a) QNM, (b) KPFM, and (c) PFM SPM results. An example topography image (i) is shown for each, as well as the relevant data channel (ii). Box plots illustrate the average properties of amorphous and crystalline nanotubes (iii). In each case, there is a statistically significant difference in the observed properties, indicating that polymer crystallization does indeed have an influence on nanoscale properties. These figures are reproduced with example line scans in the Supporting Information (Figures S11-S13).

QNM is widely used to determine the mechanical properties of nanomaterials. ${ }^{66}$ This technique operates in an intermittent contact mode, periodically indenting the sample surface with the tip and recording the deflections of the cantilever. By calibrating the stiffness of the cantilever, various mechanical properties of the sample can be inferred. Values for elastic modulus are often reported by fitting aspects of this data to indentation models, such as the Derjagin-Muller-Toropov (DMT) model. ${ }^{67}$ This model, however, assumes a spherical indenter deforming an infinite flat plane and is therefore not suitable for extracting modulus values from indentations in hollow nanotube walls (see Supporting Information S8). Instead, the deformation data field can be used to indirectly observe a relative change in modulus as a result of crystallization. Each QNM indentation occurs at a fixed load, and thus, the deformation from this load can be reliably compared between samples. FEA demonstrates that this deformation is inversely proportional to the material modulus (see Supporting Information S8), and thus the relative change in modulus as a result of crystallization can be determined.

Figure $4 \mathrm{a}$ shows the topography (i) and deformation (ii) images recorded by QNM on an individual amorphous nanotube using a peak force set-point of $100 \mathrm{nN}$. Large deformations were observed at the very edges of the nanotube. In this region, the tip side wall was in contact with the nanotube and therefore the loading geometry is not well defined. The data 

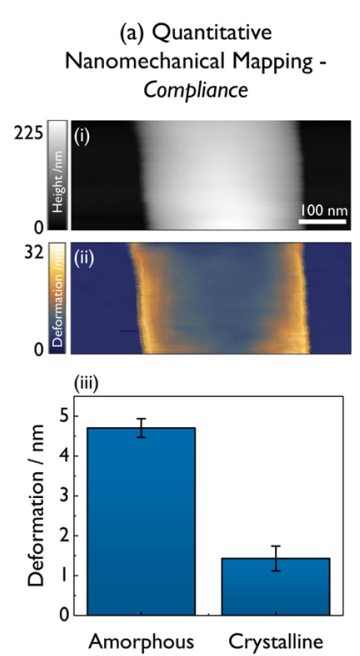

(b) Kelvin Probe Force Microscopy Surface potential
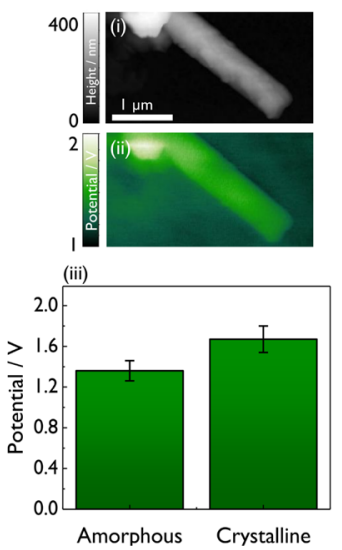

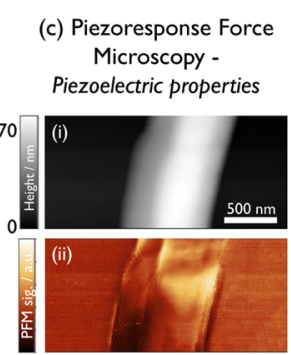

(iii)

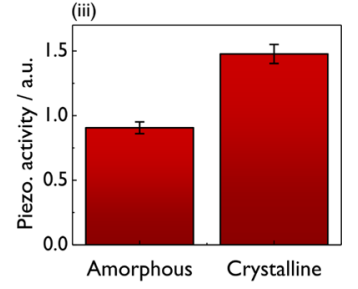

Figure 4. (a) (i) Height and (ii) deformation data from QNM of an amorphous PLLA nanotube. Part (iii) shows deformation from the center of each nanotube, averaged over the nanotube length $(p<0.01, N=3)$. (b) KPFM data showing (i) height and (ii) surface potential of crystalline nanotube. Average values in part (iii) are from the entire projected area of the nanotube $(p<0.05$, amorphous $N=3$, crystalline $N=5$ ). (c) (i) Height and (ii) lateral PFM signal from the crystalline nanotube. Box plot in (iii) represents the PFM signal gradient, which correlates to the piezoelectric coefficient $d_{14}(p<0.01, N=3)$. Error bars in all box plots represent standard deviation.

from this region are therefore difficult to interpret. It is better to consider the deformation at the center of the nanotube. This point corresponds to the loading geometry used in the simulations (see Supporting Information S8) and is more straightforward to analyze. The average central displacement of amorphous and crystalline nanotubes is shown in Figure 4a part (iii).

The deformation of the crystalline PLLA nanotubes was found to be significantly less than that of the amorphous nanotubes by a factor of $\approx 3$. Therefore, crystallization results in a three-fold increase in modulus of the PLLA nanotubes. Because the bending stiffness of each tube is linearly dependent on the Young's modulus, crystalline nanotubes appear 3 times more rigid than amorphous tubes. A similar increase in modulus was observed for bulk PLLA film samples (see Supporting Information S7). The error signal from QNM measurements can be also used to effectively highlight surface features. Some of these images are shown in Figure S14, demonstrating that while these nanotubes do have some surface roughness, it is no different between amorphous and crystalline nanotubes.

Figure 4b displays KPFM height (i) and potential (ii) values from an individual crystalline PLLA nanotube. KPFM determines the potential difference between the AFM tip and the sample surface by scanning at a fixed height above the sample and monitoring the effects of electrostatic forces on the cantilever. The box plot in Figure $4 b$ (iii) represents the average potential observed from amorphous and crystalline nanotubes. It can be seen that crystallizing the nanotubes resulted in a small but significant increase in surface potential. This increase in potential may be attributed to the more regular order of the crystalline structure and greater registry between dipoles in the polymer chains. In this regard, PLLA crystallinity has previously been shown to improve the electret properties (the ability to store charge) of the film samples. ${ }^{63}$ It is interesting to note that crystallizing bulk PLLA films resulted in a much larger increase in surface potential (see Supporting Information S9). The discrepancy between bulk and nanotube behavior can be explained by considering the molecular orientation of the PLLA nanotubes and the presence of extended chain crystals, as discussed in our previous work. ${ }^{57}$
Finally, PFM has been used to characterize any changes in piezoelectric properties that occur upon crystallization. The effect of crystallinity on piezoelectricity in polymers is not completely understood nor agreed upon. Many authors state that piezoelectric behavior is due only to the crystalline component of semicrystalline polymers. However, early work on the topic hinted that regions of aligned, but not crystalline, polymer chains may also contribute to the piezoelectric effect in polymers. ${ }^{68-70}$ This idea has been validated more recently, with careful experimental work showing that the piezoelectric effect in some polymers cannot be explained by considering the crystalline regions alone. ${ }^{71}$

Fundamentally, the requirement for piezoelectricity in PLLA is that of highly aligned polymer chains. Crystallization can be a good way to achieve this (hence the strong correlation between piezoelectric constant and crystalline fraction ${ }^{65}$ ) provided that the formation of individual crystalline domains does not remove any overall anisotropy of the sample. Figure $4 \mathrm{c}$ displays (i) height and (ii) lateral PFM signals acquired from a crystalline PLLA nanotube. The image was acquired with an oscillating potential of $8 \mathrm{~V}$ at $125 \mathrm{kHz}$. The data field presented is the amplitude of the lock-in amplifier output. ${ }^{62}$ The sawtooth profile visible in the lateral PFM signal (see Figure S13 for line scans) is characteristic of shear piezoelectricity in PLLA nanostructures, as previously demonstrated by our group. ${ }^{72}$ The gradient of this signal is directly proportional to the piezoelectric coefficient $d_{14}$. Comparing the average gradient across different samples implies that crystalline nanotubes have a piezoelectric coefficient approximately $150 \%$ greater than amorphous tubes, as shown in Figure 4c(iii).

A PFM signal was observed from amorphous nanotubes because significant molecular alignment already exists in these structures as a result of the melt-press template wetting growth method. Crystallizing the nanotubes increases the degree of this alignment, resulting in the observed increase in piezoelectric activity.

To summarize the SPM results, QNM, KPFM, and PFM have been used to investigate the properties of individual PLLA nanotubes, and how these change upon crystallization. A threefold increase in stiffness was observed when comparing 
crystalline and amorphous nanotubes. KPFM revealed that the surface potential of the nanotubes increases in a subtle manner upon crystallization. Finally, PFM verified that the nanotubes are piezoelectric, showing the same behavior as the solutiongrown PLLA nanowires described previously. ${ }^{72}$ Piezoelectric activity was observed in both amorphous and crystalline nanotubes, consistent with the observations of molecular alignment in both structures.

Importantly, the change in cellular behavior observed between amorphous and crystalline nanotubes can therefore be rationalized in terms of changes in electromechanical properties that occur during crystallization. It is well known that mechanics can have a substantial influence on the ability of fibroblasts to produce $\mathrm{ECM},{ }^{73}$ and there is a growing body of evidence that suggests electrical signals can have a significant effect on the production and composition of ECM. ${ }^{7-77}$ The differences in cell attachment between amorphous and crystalline samples agree well with these previous findings.

However, decoupling the effects of mechanics, piezoelectric properties and surface potential is challenging because all of these aspects are influenced by the crystallization process. The results from the PP NTs can help in this regard. PP is a semicrystalline but nonpiezoelectric polymer. When PP is crystallized, it will become stiffer, ${ }^{78}$ just as with PLLA, yet unlike PLLA, it does not exhibit piezoelectricity in either the amorphous or crystalline state. As such, PP NTs act as a control against the influence of stiffness in the HDF attachment assay. In addition, the variation in surface roughness between both amorphous and crystalline nanotubes of PP and PLLA shows no observed difference at the microscale, so roughness is not expected to have an influence here. ${ }^{4,46}$ It is important to stress that the absolute values of attachment cannot be directly compared between the sets of PLLA and PP nanotubes-the materials are not the same and will have different surface chemistries. The PLLA study and the PP study are internally consistent, and hence it is the relative change in cell attachment that is the relevant parameter here.

As previously discussed, the results in Figure 3 show that there is no significant difference in attachment between amorphous and crystalline PP nanotubes. This is in contrast to the case of PLLA nanotubes, where crystalline nanotubes displayed significantly greater cell attachment. Given that there is no expected or apparent difference in the microscale roughness displayed in Figure 1e(i), this suggests that it is piezoelectricity that is influencing the level of cell attachment in PLLA nanotubes. Only in the case of PLLA will crystallization result in a change in piezoelectric properties, and only in the case of PLLA does crystallization result in an increase in cell attachment. These observations suggest that it is piezoelectricity, and not mechanics, that is influencing the level of attachment. The fact that adherent cells are able to transduce the changes in piezoelectric properties under the quasistatic cell culture conditions indicates that the PLLA nanotubes are suitably "soft".

\section{CONCLUSIONS}

In this report, we demonstrate that high-aspect ratio polymeric nanostructures can be used to address the significant imbalance in stiffness between common piezoelectric materials and biological tissue, thus creating a "soft" piezoelectric surface for cell culture. The biologically derived piezoelectric polymer PLLA is an ideal material for this application because of its biocompatibility, and perhaps more importantly, because of its shear piezoelectric properties. PLLA nanotubes produced via melt-press template wetting have been found to possess the correct polymer chain orientation to express the piezoelectric properties of PLLA in bending configuration. HDF cells cultured onto the nanotube surfaces were observed to be deforming the nanostructures, thus validating the idea of creating a "soft" piezoelectric surface from nanostructured piezoelectric PLLA for biological studies. Cells were found to attach readily to PLLA nanotubes but not to films of the same material. Crystalline PLLA nanotubes displayed greater attachment compared to amorphous structures, almost equal to TCP despite the lack of any surface treatment or adhesive factors.

SPM investigations demonstrated that this difference in behavior was as a result of significant changes to the electromechanical properties of the polymer occurring upon crystallization. KPFM revealed that the surface potential of the nanotubes increases in a subtle way upon crystallization. Using PF-QNM, a three-fold increase in stiffness was observed between crystalline and amorphous nanotubes. Finally, PFM verified that the nanotubes are piezoelectric, with an increase in activity between amorphous and crystalline nanotubes. Control experiments with nonpiezoelectric nanotubes indicated that the changes in piezoelectricity are largely responsible for the changes in cell behavior. This outcome is only possible because of the nanostructured surface-cells attached to these nanotubes can only transduce changes in piezoelectric properties through mechanical interactions, and meaningful mechanical interactions are only possible because of low effective stiffness afforded using the high-aspect ratio nanostructures.

\section{METHODS}

FEA Simulations. COMSOL Multiphysics 5.3a was used for all FEA simulations. The PLLA nanotube length, radius, and wall thickness were varied systematically using the parametric sweep function. Material parameters used can be found in Table S2. A quadrilateral mesh was swept along the axial direction of the nanotube. The maximum mesh element size was one-half of the wall thickness, validated with a convergence test (see Supporting Information S4). Likewise, the dimensions of the mesh of elements along the length of the tube were subjected to a convergence test, with $50 \mathrm{~nm}$ elements being found to be satisfactory. To model the piezoelectric and mechanical response, a $1 \mathrm{nN}$ load was applied perpendicularly to the top face of the tube. The bottom face was rigidly fixed and set to the electrical ground. Modeling the entire $30 \mu \mathrm{m}$ length observed experimentally is impractical because of the high aspect ratio and high geometric nonlinearity of the computation, but the results from these simulations can nonetheless be transferred.

Nanotube Fabrication. Pellets of PLLA (Lactel B6002-2, SigmaAldrich) were used to fabricate the nanotubes. The melt-press template wetting method of fabrication has been described previously. ${ }^{57}$ Briefly, pellets of the polymer are heated to c. $190{ }^{\circ} \mathrm{C}$ and pressed into a nanoporous membrane of anodized aluminium oxide (AAO). The molten polymer enters the pores in the template, coating the walls to form nanotubes. The resulting nanotubes have diameter $305 \pm 24 \mathrm{~nm}$ and wall thickness $56 \pm 10 \mathrm{~nm} .{ }^{57}$ Crystalline samples were heat-treated in an oven at $120{ }^{\circ} \mathrm{C}$ for $1 \mathrm{~h}$, which resulted in samples with approximately $50 \%$ crystallinity as assessed by X-ray diffraction (XRD) ${ }^{57}$ Amorphous samples were not heat-treated and displayed no measurable XRD peaks. The template material was removed by etching in phosphoric acid ( $40 \% \mathrm{v} / \mathrm{v}$ in water) for $3 \mathrm{~h}$. After etching, samples were washed five times in water and once in ethanol. Analogous flat films were produced in an identical manner, except without the AAO template.

PP nanotubes were fabricated using the same melt-press template wetting method. Pellets of isotactic PP (Goodfellow) were heated to $250^{\circ} \mathrm{C}$ and pressed into the AAO membranes. Isotactic PP crystallizes readily, and cooling in air after pressing is insufficient to produce 
amorphous samples. To avoid crystallization, samples were quenched to room temperature with water bath. Crystalline samples were produced via a heat treatment at $160{ }^{\circ} \mathrm{C}$ for $1 \mathrm{~h}$. XRD patterns of quenched and annealed samples can be found in the Figure S15. Crystalline samples had $\approx 40 \%$ (volume) crystalline fraction, while amorphous samples were less than $5 \%$ crystalline.

SPM Characterisation. For SPM measurements, the nanotextured film was sonicated in isopropyl alcohol for $10 \mathrm{~min}$ to dislodge some of the nanotubes. The nanotube suspension was then cast onto the palladium-coated silicon wafer and mounted onto a magnetic AFM mount with silver paint. All SPM characterization was performed using a Bruker MultiMode 8 atomic force microscope with a MESP-RC-V2 probe (Bruker). KPFM scans were performed at a scan rate of $0.5 \mathrm{~Hz}$ and lift height $50 \mathrm{~nm}$. The potential values from KPFM were applied as a dc bias during PFM to mitigate artefacts from electrostatic potential. ${ }^{79}$ PFM scans were carried out with an oscillating potential at $125 \mathrm{kHz}$ and an $8 \mathrm{~V}$ zero-to-peak amplitude in a similar manner to what was described previously. ${ }^{72}$ Scan rate was $0.1 \mathrm{~Hz}$. The PFM output was not calibrated and the lock-in amplifier output is presented in arbitrary units. For QNM, the cantilever sensitivity was calibrated by performing a series of ramps on a sapphire sample (SAPPHIRE-12M, Bruker). A thermal tune was then used to determine the cantilever spring constant. A peak-force set point of $100 \mathrm{nN}$ was used for all scans.

Cell Testing. Cell Preparation. HDF (Sigma-Aldrich) were cultured under standard tissue culture conditions $\left(37^{\circ} \mathrm{C}, 5 \% \mathrm{CO}_{2}\right)$ in T75 flasks with growth media consisting of Dulbecco's modified Eagle's medium (Sigma-Aldrich) with $10 \%(\mathrm{v} / \mathrm{v})$ fetal bovine serum (Sigma-Aldrich) and $1 \%(\mathrm{v} / \mathrm{v})$ streptomycin/penicillin (SigmaAldrich). Cells were prepared for experiments by detaching from the cell culture flask using $0.05 \%(\mathrm{w} / \mathrm{v})$ trypsin $/ 0.02 \%(\mathrm{w} / \mathrm{v})$ EDTA (Sigma-Aldrich) and resuspended in growth media at a known density as measured by manual counting with a haemocytometer.

Sample Preparation. An $8 \mathrm{~mm}$ biopsy punch was used to cut samples from the nanotextured and flat films for cell testing. Six independent films were prepared for each condition, and one sample was cut for cell testing from each film. After cutting, the samples were each sonicated in ethanol for $30 \mathrm{~s}$. Samples were placed into separate wells of a 48 well plate (CytoOne). When growth media was added, it was often found that samples would float as a result of gas bubbles nucleating on the rough sample surface. To prevent this, a thin neodymium magnet was fixed to the underside of the plate and small gold-coated neodymium magnets were placed on top of the samples in each well $(1.5 \times 0.75 \mathrm{~mm}$ N52 gold plated disc, Gaussboys, USA). The small magnetic attraction through the base of the plate was sufficient to prevent the samples from floating. To ensure that the presence of the magnets did not influence the cell behavior, magnet pairs were also added to wells containing only TCP (i.e., without sample material) and compared to a TCP positive control. No change in cell behavior was observed between magnetic wells and the positive control-see Supporting Information S10.

Cell Attachment Assay. A lactate dehydrogenase assay was used to determine cell attachment. A $500 \mu \mathrm{L}$ volume of cells at a density of $25 \times$ $10^{4}$ cells $/ \mathrm{mL}$ was added to each well containing samples, alongside TCP controls and the TCP + magnet control wells. The entire plate was incubated at $37^{\circ} \mathrm{C}$ and $5 \% \mathrm{CO}_{2}$ for $14 \mathrm{~h}$. After this incubation period, the growth media was removed, and all wells were gently washed with 2 $\times 500 \mu \mathrm{L}$ phosphate buffered saline (PBS) solution to remove loosely bound cells. The samples were then transferred to a new 48 well plate. Cells were lysed using a $200 \mu \mathrm{L}$ volume of $2 \%(\mathrm{v} / \mathrm{v})$ Triton X-100 in deionised (DI) water at room temperature for $2 \mathrm{~h}$. A $100 \mu \mathrm{L}$ volume of the lysate was then transferred to a new 96 well plate, and $100 \mu \mathrm{L}$ of $\mathrm{LDH}$ substrate (Sigma-Aldrich) was also added to each well. This was left for $10 \mathrm{~min}$ at room temperature, after which the absorbance was measured at $490 \mathrm{~nm}$ using a plate-reader (SPECTROStar Nano, BMG Labtech).

Converting the values of absorbance back to percentage attachment was achieved by including a set of known cell density calibration wells on each plate. In these wells, a known percentage of cells was added- 0 , $10,25,50$, and $100 \%$ - with the remainder of the volume being made up with growth media to $500 \mu \mathrm{L}$. These wells were washed very gently with $1 \times 500 \mu \mathrm{L}$ PBS after incubation to minimize the removal of cells. The values of absorbance were then plotted against the percentage of cells added and fitted to the linear model. This linear relationship was then used to convert values of absorbance into a percentage attachment.

Data analysis was performed in Origin 2016. Wells known to be subject to pipetting errors were omitted from the analysis. Significance was assessed using a two sample $t$-test assuming unequal variances. The box represents standard error in mean, and whiskers represent minimum and maximum values.

Rhodamine and 4',6-Diamidino-2-phenylindole Staining. Samples were prepared as mentioned above. A $500 \mu \mathrm{L}$ volume of cells at a density of $50 \times 10^{4}$ cells $/ \mathrm{mL}$ was added to each well. The entire plate was incubated at $37^{\circ} \mathrm{C}$ and $5 \% \mathrm{CO}_{2}$ for $72 \mathrm{~h}$. Samples were then fixed using 5\% (v/v) glutaraldehyde (Sigma-Aldrich) in PBS for $20 \mathrm{~min}$. Cells were lysed for $1.5 \mathrm{~h}$ with $0.5 \%(\mathrm{v} / \mathrm{v})$ Triton-X solution in PBS for $5 \mathrm{~min}$ and stained with $0.1 \%(\mathrm{v} / \mathrm{v})$ rhodamine phalloidin in PBS (Sigma-Aldrich) for $45 \mathrm{~min}$. After staining, samples were washed with DI water and stained with $0.01 \%$ (v/v) 4',6-diamidino-2-phenylindole (DAPI) (Sigma-Aldrich) solution in DI water for $5 \mathrm{~min}$. Following another washing with DI water, samples were observed using a Zeiss Observer Z1 fluorescent microscope (Carl Zeiss Ltd.).

Live Dead Staining. Samples were prepared as mentioned above. A $500 \mu \mathrm{L}$ volume of cells at a density of $25 \times 10^{4}$ cells $/ \mathrm{mL}$ was added to each well. The entire plate was incubated at $37^{\circ} \mathrm{C}$ and $5 \% \mathrm{CO}_{2}$ for $72 \mathrm{~h}$. Before staining, the cell media was removed and samples were washed with PBS. Briefly, cells were then stained with $2 \mu \mathrm{M}$ calcein AM and 4 $\mu \mathrm{M}$ ethidium homodimer- 1 in PBS for $30 \mathrm{~min}$ in a humidified incubator with $5 \% \mathrm{CO}_{2}$ at $37{ }^{\circ} \mathrm{C}$. Imaging was performed immediately after staining.

SEM Sample Preparation. Samples were prepared as mentioned above. A $500 \mu \mathrm{L}$ volume of cells at a density of $25 \times 10^{4}$ cells $/ \mathrm{mL}$ was added to each well. The entire plate was incubated at $37^{\circ} \mathrm{C}$ and $5 \% \mathrm{CO}_{2}$ for $72 \mathrm{~h}$. After incubation, the samples were fixed with $5 \%(\mathrm{v} / \mathrm{v})$ glutaraldehyde (Sigma-Aldrich) in PBS for $20 \mathrm{~min}$. The samples were then immersed in serial dilutions of water/ethanol, for $1 \mathrm{~min}$ at a time followed by immersion in serial dilutions of ethanol/hexamethyldisilazane (HMDS, Sigma-Aldrich) for 5 min each, finally allowing any remaining HMDS to evaporate. Once dry, samples were sputter-coated with palladium and imaged using a Hitachi TM3030 desktop scanning electron microscope.

\section{ASSOCIATED CONTENT}

\section{Supporting Information}

The Supporting Information is available free of charge at https://pubs.acs.org/doi/10.1021/acsabm.0c00012.

Derivation of the effective stiffness ratio; importance of polymer chain alignment in piezoelectric polymers; parametric sweep of the tube geometry; convergence of FEA modeling; additional SEM images of cell/nanotube interaction; upper and lower bounds of bending stiffness and effective shear modulus; QNM of PLLA films; FEA modeling of nanotube side wall deflection; KPFM of PLLA films; magnetic well attachment control; extended QNM data; extended KPFM data; extended PFM data; PeakForce QNM of PLLA nanotubes to the observed surface morphology; attachment of HDFs to polypropylene nanotubes; and material parameters for FEA modeling (PDF)

\section{AUTHOR INFORMATION}

\section{Corresponding Author}

Sohini Kar-Narayan - Department of Materials Science \& Metallurgy, University of Cambridge, Cambridge CB3 OFS, U.K.; ○ orcid.org/0000-0002-8151-1616; Email: sk568@ cam.ac.uk 


\section{Authors}

Michael Smith - Department of Materials Science \& Metallurgy, University of Cambridge, Cambridge CB3 OFS, U.K.

Thomas Chalklen - Department of Materials Science \& Metallurgy, University of Cambridge, Cambridge CB3 OFS, U.K.

Cathrin Lindackers - Department of Materials Science \& Metallurgy, University of Cambridge, Cambridge CB3 OFS, U.K.

Yonatan Calahorra - Department of Materials Science \& Metallurgy, University of Cambridge, Cambridge CB3 OFS,

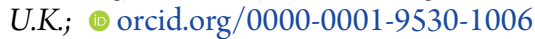

Caitlin Howe - Department of Materials Science \& Metallurgy, University of Cambridge, Cambridge CB3 OFS, U.K.

Alkausil Tamboli - Department of Materials Science \& Metallurgy, University of Cambridge, Cambridge CB3 OFS, U.K.

Daniel V. Bax - Department of Materials Science \& Metallurgy, University of Cambridge, Cambridge CB3 OFS, U.K.

David J. Barrett - Department of Materials Science \& Metallurgy, University of Cambridge, Cambridge CB3 OFS, U.K.

Ruth E. Cameron - Department of Materials Science \& Metallurgy, University of Cambridge, Cambridge CB3 OFS, U.K.

Serena M. Best - Department of Materials Science \& Metallurgy, University of Cambridge, Cambridge CB3 OFS, U.K.

Complete contact information is available at:

https://pubs.acs.org/10.1021/acsabm.0c00012

\section{Author Contributions}

M.S. devised the experiments and analyzed the results, and S.K.N. guided the work. Samples were prepared by M.S., C.L., T.C., A.T., and C.L. Y.C. assisted with all aspects of SPM, C.L. carried out some of the SPM characterization. S.M.B., R.E.C., D.J.B., and D.V.B. facilitated all aspects of cell culture. M.S., T.C., and S.K.-N. wrote the manuscript.

\section{Funding}

S.K.-N. and M.S. are grateful for the financial support from the European Research Council through an ERC Starting Grant (grant no. ERC-2014-STG-639526, NANOGEN) and the Biotechnology and Biological Sciences Research Council (BB/ R022283/1). R.E.C, S.M.B, D.J.B., and D.V.B acknowledge funding from EPSRC grant EP/N019938/1 (Established Career Fellowship grant). M.S. acknowledges studentship funding from the Cambridge Commonwealth, European and International Trust.

\section{Notes}

The authors declare no competing financial interest.

Supporting data for this paper is available at the DSpace@ Cambridge data repository (https://doi.org/10.17863/CAM. 50865).

\section{ABBREVIATIONS}

HDF, human dermal fibroblast; NT, nanotube; PLLA, poly-Llactic acid; PP, polypropylene; SPM, scanning probe microscopy; ECM, extracellular matrix

\section{REFERENCES}

(1) Solon, J.; Levental, I.; Sengupta, K.; Georges, P. C.; Janmey, P. A. Fibroblast Adaptation and Stiffness Matching to Soft Elastic Substrates. Biophys. J. 2007, 93, 4453-4461.

(2) Janmey, P. A.; Winer, J. P.; Murray, M. E.; Wen, Q. The Hard Life of Soft Cells. Cell Motil. Cytoskeleton 2009, 66, 597-605.

(3) Fu, J.; Wang, Y.-K.; Yang, M. T.; Desai, R. A.; Yu, X.; Liu, Z.; Chen, C. S. Mechanical Regulation of Cell Function with Geometrically Modulated Elastomeric Substrates. Nat. Methods 2010, 7, 733-736.
(4) Buxboim, A.; Discher, D. E. Stem Cells Feel the Difference. Nat. Methods 2010, 7, 695-697.

(5) Thrivikraman, G.; Boda, S. K.; Basu, B. Unraveling the Mechanistic Effects of Electric Field Stimulation towards Directing Stem Cell Fate and Function: A Tissue Engineering Perspective. Biomaterials 2018, 150, 60-86.

(6) Hoffman, B. D.; Crocker, J. C. Cell Mechanics: Dissecting the Physical Responses of Cells to Force. Annu. Rev. Biomed. Eng. 2009, 11, 259-288.

(7) Vogel, V.; Sheetz, M. Local Force and Geometry Sensing Regulate Cell Functions. Nat. Rev. Mol. Cell Biol. 2006, 7, 265-275.

(8) Iskratsch, T.; Wolfenson, H.; Sheetz, M. P. Appreciating Force and Shape-the Rise of Mechanotransduction in Cell Biology. Nat. Rev. Mol. Cell Biol. 2014, 15, 825-833.

(9) Moeendarbary, E.; Harris, A. R. Cell Mechanics: Principles, Practices, and Prospects. Wiley Interdiscip. Rev.: Syst. Biol. Med. 2014, 6, 371-388.

(10) Tarabella, G.; Mahvash Mohammadi, F.; Coppedè, N.; Barbero, F.; Iannotta, S.; Santato, C.; Cicoira, F. New Opportunities for Organic Electronics and Bioelectronics: Ions in Action. Chem. Sci. 2013, 4, 1395-1409.

(11) Owens, R.; Kjall, P.; Richter-Dahlfors, A.; Cicoira, F. Organic Bioelectronics - Novel Applications in Biomedicine. Biochim. Biophys. Acta, Gen. Subj. 2013, 1830, 4283-4285.

(12) Svennersten, K.; Larsson, K. C.; Berggren, M.; Richter-Dahlfors, A. Organic Bioelectronics in Nanomedicine. Biochim. Biophys. Acta, Gen. Subj. 2011, 1810, 276-285.

(13) Moulton, S. E.; Higgins, M. J.; Kapsa, R. M. I.; Wallace, G. G. Organic Bionics: A New Dimension in Neural Communications. Adv. Funct. Mater. 2012, 22, 2003-2014.

(14) Ergir, E.; Bachmann, B.; Redl, H.; Forte, G.; Ertl, P. Small Force, Big Impact: Next Generation Organ-on-a-Chip Systems Incorporating Biomechanical Cues. Front. Physiol. 2018, 9, 1417.

(15) Modena, M. M.; Chawla, K.; Misun, P. M.; Hierlemann, A. Smart Cell Culture Systems: Integration of Sensors and Actuators into Microphysiological Systems. ACS Chem. Biol. 2018, 13, 1767-1784.

(16) Fukada, E. Piezoelectricity in Polymers and Biological Materials. Ultrasonics 1968, 6, 229-234.

(17) Fukada, E. Bioelectrets and Biopiezoelectricity. IEEE Trans. Electr. Insul. 1992, 27, 813-819.

(18) Shamos, M. H.; Lavine, L. S. Piezoelectricity as a Fundamental Property of Biological Tissues. Nature 1967, 213, 267-269.

(19) Guerin, S.; Stapleton, A.; Chovan, D.; Mouras, R.; Gleeson, M.; McKeown, C.; Noor, M. R.; Silien, C.; Rhen, F. M. F.; Kholkin, A. L.; Liu, N.; Soulimane, T.; Tofail, S. A. M.; Thompson, D. Control of Piezoelectricity in Amino Acids by Supramolecular Packing. Nat. Mater. 2018, 17, 180-186.

(20) Ning, C.; Zhou, Z.; Tan, G.; Zhu, Y.; Mao, C. Electroactive Polymers for Tissue Regeneration: Developments and Perspectives. Prog. Polym. Sci. 2018, 81, 144-162.

(21) Marino, A.; Genchi, G. G.; Sinibaldi, E.; Ciofani, G. Piezoelectric Effects of Materials on Bio-Interfaces. ACS Appl. Mater. Interfaces 2017, 9, 17663-17680.

(22) Marino, A.; Genchi, G. G.; Mattoli, V.; Ciofani, G. Piezoelectric Nanotransducers: The Future of Neural Stimulation. Nano Today 2017, 14, 9-12.

(23) Rajabi, A. H.; Jaffe, M.; Arinzeh, T. L. Piezoelectric Materials for Tissue Regeneration: A Review. Acta Biomater. 2015, 24, 12-23.

(24) Tandon, B.; Blaker, J. J.; Cartmell, S. H. Piezoelectric Materials as Stimulatory Biomedical Materials and Scaffolds for Bone Repair. Acta Biomater. 2018, 73, 1-20.

(25) Ribeiro, C.; Sencadas, V.; Correia, D. M.; Lanceros-Méndez, S. Piezoelectric Polymers as Biomaterials for Tissue Engineering Applications. Colloids Surf., B 2015, 136, 46-55.

(26) Laurenti, M.; Cauda, V. ZnO Nanostructures for Tissue Engineering Applications. Nanomaterials 2017, 7, 374.

(27) Chorsi, M. T.; Curry, E. J.; Chorsi, H. T.; Das, R.; Baroody, J.; Purohit, P. K.; Ilies, H.; Nguyen, T. D. Piezoelectric Biomaterials for Sensors and Actuators. Adv. Mater. 2018, 31, 1802084. 
(28) Tang, Y.; Wu, C.; Wu, Z.; Hu, L.; Zhang, W.; Zhao, K. Fabrication and in Vitro Biological Properties of Piezoelectric Bioceramics for Bone Regeneration. Sci. Rep. 2017, 7, 43360.

(29) Wen, J.; Liu, M. Piezoelectric Ceramic (PZT) Modulates Axonal Guidance Growth of Rat Cortical Neurons via RhoA, Rac1, and Cdc42 Pathways. J. Mol. Neurosci. 2014, 52, 323-330.

(30) Guo, H.-F.; Li, Z.-S.; Dong, S.-W.; Chen, W.-J.; Deng, L.; Wang, Y.-F.; Ying, D.-J. Piezoelectric PU/PVDF Electrospun Scaffolds for Wound Healing Applications. Colloids Surf., B 2012, 96, 29-36.

(31) Royo-Gascon, N.; Wininger, M.; Scheinbeim, J. I.; Firestein, B. L.; Craelius, W. Piezoelectric Substrates Promote Neurite Growth in Rat Spinal Cord Neurons. Ann. Biomed. Eng. 2013, 41, 112-122.

(32) Wang, A.; Hu, M.; Zhou, L.; Qiang, X. Self-Powered WellAligned P(VDF-TrFE) Piezoelectric Nanofiber Nanogenerator for Modulating an Exact Electrical Stimulation and Enhancing the Proliferation of Preosteoblasts. Nanomaterials 2019, 9, 349.

(33) Wu, S.; Chen, M.-S.; Maurel, P.; Lee, Y.-s.; Bunge, M. B.; Arinzeh, T. L. Aligned Fibrous PVDF-TrFE Scaffolds with Schwann Cells Support Neurite Extension and Myelination in Vitro. J. Neural. Eng. 2018, 15, 056010

(34) Mota, C.; Labardi, M.; Trombi, L.; Astolfi, L.; D’Acunto, M.; Puppi, D.; Gallone, G.; Chiellini, F.; Berrettini, S.; Bruschini, L.; Danti, S. Design, Fabrication and Characterization of Composite Piezoelectric Ultrafine Fibers for Cochlear Stimulation. Mater. Des. 2017, 122, $206-$ 219.

(35) Genchi, G. G.; Sinibaldi, E.; Ceseracciu, L.; Labardi, M.; Marino, A.; Marras, S.; De Simoni, G.; Mattoli, V.; Ciofani, G. UltrasoundActivated Piezoelectric P(VDF-TrFE)/Boron Nitride Nanotube Composite Films Promote Differentiation of Human SaOS-2 Osteoblast-like Cells. Nanomed. Nanotechnol. Biol. Med. 2017, 14, 2421.

(36) Hoop, M.; Chen, X.-Z.; Ferrari, A.; Mushtaq, F.; Ghazaryan, G.; Tervoort, T.; Poulikakos, D.; Nelson, B.; Pané, S. Ultrasound-Mediated Piezoelectric Differentiation of Neuron-like PC12 Cells on PVDF Membranes. Sci. Rep. 2017, 7, 4028.

(37) Ribeiro, C.; Pärssinen, J.; Sencadas, V.; Correia, V.; Miettinen, S.; Hytönen, V. P.; Lanceros-Méndez, S. Dynamic Piezoelectric Stimulation Enhances Osteogenic Differentiation of Human Adipose Stem Cells. J. Biomed. Mater. Res., Part A 2015, 103, 2172-2175.

(38) Rodriguez, M. L.; McGarry, P. J.; Sniadecki, N. J. Review on Cell Mechanics: Experimental and Modeling Approaches. Appl. Mech. Rev. 2013, 65, 060801.

(39) Vogel, S. Comparative Biomechanics: Life's Physical World, 2nd ed.; Princeton University Press, 2013.

(40) Zhang, Q. M.; Jianzhong Zhao, J. Electromechanical Properties of Lead Zirconate Titanate Piezoceramics under the Influence of Mechanical Stresses. IEEE Trans. Ultrason. Eng. 1999, 46, 1518-1526.

(41) Desai, A. V.; Haque, M. A. Mechanical Properties of $\mathrm{ZnO}$ Nanowires. Sens. Actuators, A 2007, 134, 169-176.

(42) Vinogradov, A.; Holloway, F. Electro-Mechanical Properties of the Piezoelectric Polymer PVDF. Ferroelectrics 1999, 226, 169-181.

(43) Murillo, G.; Blanquer, A.; Vargas-Estevez, C.; Barrios, L.; Ibáñez, E.; Nogués, C.; Esteve, J. Electromechanical Nanogenerator-Cell Interaction Modulates Cell Activity. Adv. Mater. 2017, 29, 1605048.

(44) Ribeiro, C.; Sencadas, V.; Areias, A. C.; Gama, F. M.; LancerosMéndez, S. Surface Roughness Dependent Osteoblast and Fibroblast Response on Poly(1 -Lactide) Films and Electrospun Membranes. J. Biomed. Mater. Res., Part A 2015, 103, 2260-2268.

(45) Areias, A. C.; Ribeiro, C.; Sencadas, V.; Garcia-Giralt, N.; DiezPerez, A.; Gómez Ribelles, J. L.; Lanceros-Méndez, S. Influence of Crystallinity and Fiber Orientation on Hydrophobicity and Biological Response of Poly(l-Lactide) Electrospun Mats. Soft Matter 2012, 8, 5818.

(46) Park, A.; Cima, L. G. In Vitro Cell Response to Differences in Poly-L-Lactide Crystallinity. J. Biomed. Mater. Res. 1996, 31, 117-130.

(47) Degirmenbasi, N.; Ozkan, S.; Kalyon, D. M.; Yu, X. Surface Patterning of Poly(L-Lactide) upon Melt Processing: In Vitro Culturing of Fibroblasts and Osteoblasts on Surfaces Ranging from Highly Crystalline with Spherulitic Protrusions to Amorphous with
Nanoscale Indentations. J. Biomed. Mater. Res., Part A 2009, 88A, 94104.

(48) Harrison, J. S.; Ounaies, Z. Piezoelectric Polymers. Encyclopedia of Polymer Science and Technology; American Cancer Society, 2001; Vol. 8.

(49) Jamshidian, M.; Tehrany, E. A.; Imran, M.; Jacquot, M.; Desobry, S. Poly-Lactic Acid: Production, Applications, Nanocomposites, and Release Studies. Compr. Rev. Food Sci. Food Saf. 2010, 9, 552-571.

(50) Ochiai, T.; Fukada, E. Electromechanical Properties of Poly-LLactic Acid. Jpn. J. Appl. Phys. 1998, 37, 3374-3376.

(51) Middleton, J. C.; Tipton, A. J. Synthetic Biodegradable Polymers as Orthopedic Devices. Biomaterials 2000, 21, 2335-2346.

(52) Datta, R.; Henry, M. Lactic acid: recent advances in products, processes and technologies - a review. J. Chem. Technol. Biotechnol. 2006, 81, 1119-1129.

(53) Ikada, Y.; Shikinami, Y.; Hara, Y.; Tagawa, M.; Fukada, E. Enhancement of Bone Formation by Drawn Poly(L-Lactide). J. Biomed. Mater. Res. 1996, 30, 553-558.

(54) Ando, M.; Takeshima, S.; Ishiura, Y.; Ando, K.; Onishi, O. Piezoelectric Antibacterial Fabric Comprised of Poly(l-Lactic Acid) Yarn. Jpn. J. Appl. Phys. 2017, 56, 10PG01.

(55) Tajitsu, Y. Smart Piezoelectric Fabric and Its Application to Control of Humanoid Robot. Ferroelectrics 2016, 499, 36-46.

(56) Tajitsu, Y. Piezoelectric Poly-L-Lactic Acid Fabric and Its Application to Control of Humanoid Robot. Ferroelectrics 2017, 515, $44-58$.

(57) Smith, M.; Lindackers, C.; McCarthy, K.; Kar-Narayan, S. Enhanced Molecular Alignment in Poly- 1 -Lactic Acid Nanotubes Induced via Melt-Press Template-Wetting. Macromol. Mater. Eng. 2019, 304, 1800607.

(58) Tsuji, H.; Nakahara, K.; Ikarashi, K. Poly(L-Lactide), 8. HighTemperature Hydrolysis of Poly(L-Lactide) Films with Different Crystallinities and Crystalline Thicknesses in Phosphate-Buffered Solution. Macromol. Mater. Eng. 2001, 286, 398-406.

(59) Choi, Y. S.; Kim, S. K.; Williams, F.; Calahorra, Y.; Elliott, J. A.; Kar-Narayan, S. The Effect of Crystal Structure on the Electromechanical Properties of Piezoelectric Nylon-11 Nanowires. Chem. Commun. 2018, 54, 6863-6866.

(60) Melitz, W.; Shen, J.; Kummel, A. C.; Lee, S. Kelvin Probe Force Microscopy and Its Application. Surf. Sci. Rep. 2011, 66, 1-27.

(61) Pittenger, B.; Erina, N.; Su, C. Mechanical Property Mapping at the Nanoscale Using PeakForce QNM Scanning Probe Technique. In Nanomechanical Analysis of High Performance Materials; Tiwari, A., Ed.; Springer Netherlands: Dordrecht, 2014; pp 31-51.

(62) Denning, D.; Guyonnet, J.; Rodriguez, B. J. Applications of Piezoresponse Force Microscopy in Materials Research: From Inorganic Ferroelectrics to Biopiezoelectrics and Beyond. Int. Mater. Rev. 2016, 61, 46-70.

(63) Guzhova, A. A.; Galikhanov, M. F.; Kuznetsova, N. V.; Petrov, V. A.; Khairullin, R. Z. Effect of Polylactic Acid Crystallinity on Its Electret Properties. AIP Conf. Proc. 2016, 1767, 020009.

(64) Garlotta, D. Literature Review of Poly(Lactic Acid). J. Polym. Environ. 2001, 9, 63-84.

(65) Lovell, C. S.; Fitz-Gerald, J. M.; Park, C. Decoupling the Effects of Crystallinity and Orientation on the Shear Piezoelectricity of Polylactic Acid. J. Polym. Sci., Part B: Polym. Phys. 2011, 49, 1555-1562.

(66) Lawrence, J. G.; Berhan, L. M.; Nadarajah, a. Elastic Properties and Morphology of Individual Carbon Nanofibers. ACS Nano 2008, 2, $1230-1236$

(67) Derjaguin, B. V.; Muller, V. M.; Toporov, Y. P. Effect of Contact Deformations on the Adhesion of Particles. J. Colloid Interface Sci. 1975, 53, 314-326.

(68) Fukada, E. Piezoelectricity and Pyroelectricity of Biopolymers. In Ferroelectric Polymers: Chemistry, Physics and Applications; Nalwa, H. S., Ed.; CRC Press, 1995; pp 393-434.

(69) Fukada, E. History and Recent Progress in Piezoelectric Polymers. IEEE Trans. Ultrason. Eng. 2000, 47, 1277-1290.

(70) Fukada, E. Recent Developments of Polar Piezoelectric Polymers. IEEE Trans. Dielectr. Electr. Insul. 2006, 13, 1110-1119. 
(71) Katsouras, I.; Asadi, K.; Li, M.; van Driel, T. B.; Kjær, K. S.; Zhao, D.; Lenz, T.; Gu, Y.; Blom, P. W. M.; Damjanovic, D.; Nielsen, M. M.; de Leeuw, D. M. The Negative Piezoelectric Effect of the Ferroelectric Polymer Poly(Vinylidene Fluoride). Nat. Mater. 2016, 15, 78-84.

(72) Smith, M.; Calahorra, Y.; Jing, Q.; Kar-Narayan, S. Direct Observation of Shear Piezoelectricity in Poly-l-Lactic Acid Nanowires. APL Mater. 2017, 5, 074105.

(73) Chiquet, M.; Renedo, A. S.; Huber, F.; Flück, M. How Do Fibroblasts Translate Mechanical Signals into Changes in Extracellular Matrix Production? Matrix Biol. 2003, 22, 73-80.

(74) Nguyen, H. T.; Wei, C.; Chow, J. K.; Nguy, L.; Nguyen, H. K.; Schmidt, C. E. Electric Field Stimulation through a Substrate Influences Schwann Cell and Extracellular Matrix Structure. J. Neural. Eng. 2013, $10,046011$.

(75) Wang, Y.; Rouabhia, M.; Zhang, Z. Pulsed Electrical Stimulation Benefits Wound Healing by Activating Skin Fibroblasts through the TGF $\beta 1 /$ ERK/NF-KB Axis. Biochim. Biophys. Acta, Gen. Subj. 2016, 1860, 1551-1559.

(76) Hunckler, J.; de Mel, A. A Current Affair: Electrotherapy in Wound Healing. J. Multidiscip. Healthc. 2017, 10, 179-194.

(77) Nguyen, E. B.; Wishner, J.; Slowinska, K. The Effect of Pulsed Electric Field on Expression of ECM Proteins: Collagen, Elastin, and MMP1 in Human Dermal Fibroblasts. J. Electroanal. Chem. 2018, 812, 265-272.

(78) Menyhard, A.; Suba, P.; Laszlo, Z.; Fekete, H. M.; Mester, A. O.; Horvath, Z.; Voros, G.; Varga, J.; Moczo, J. Direct Correlation between Modulus and the Crystalline Structure in Isotactic Polypropylene. eXPRESS Polym. Lett. 2015, 9, 308-320.

(79) Kim, S.; Seol, D.; Lu, X.; Alexe, M.; Kim, Y. Electrostatic-Free Piezoresponse Force Microscopy. Sci. Rep. 2017, 7, 41657. 